\title{
Full mouth rehabilitation with tooth implant connection prosthesis: A case report with follow up
}

\author{
Dhruv Anand ${ }^{1, *}$, Rahul Sharma ${ }^{2}$, Devanshi Yadav Anand ${ }^{3}$ \\ ${ }^{\mathbf{1}}$ Lecturer, Dept. of Prosthodontics, School of Dental Sciences, Sharda University, Greater Noida, Uttar Pradesh, India, ${ }^{\mathbf{2}, \mathbf{P} P r i v a t e}$ \\ Practioner
}

*Corresponding Author:

Email: diadhruv@gmail.com

\begin{abstract}
A Dental prosthesis that involves the combination of dental implants and natural teeth to replace missing teeth can not only increase the retention of the prosthesis but also provide good stability and aesthetic outcomes in most of the situations. However, prudent incorporation of the biomechanical principles is necessary for the design and construction of the prosthesis. Due to the anatomical differences in the functioning of tooth and an implant, certain issues can arise in the long-term success of the treatment that involves tooth-implant connection prosthesis.
\end{abstract}

Keywords: Fixed partial dentures, Rigid connection, Combination prosthesis, Implants, Full mouth rehabilitation.

\section{Introduction}

Since many years dental Implant treatment has emerged as a universally accepted treatment modality by the clinicians worldwide.

It has been proved that long term success rate of dental implant is more than $90 \%$ for treatment of both completely and partially edentulous patients.

However, the combination of natural teeth and dental implants to fabricate a prosthesis still remains a controversial issue as the survival rate of such prosthesis is lower compared with solely Implant supported prosthesis or tooth supported fixed dental prosthesis.

The biomechanics of a dental Implant is different from that of a natural tooth, as dental implants integrate by the process of osseointegration that allows them to fuse with the supporting bone. Whereas, in case of natural dentition, the surrounding periodontal ligament allows physiological mobilisation of the teeth.

Dental Implants \& natural teeth behave differently when exposed to masticatory load due to the difference in their biomechanics. However, sometimes in an unavoidable situation, an ideal prosthesis that involves only teeth cannot be given.

Long term success of the Implant supported prosthesis is abundant whereas, limited data is available on the long term prognosis of a combination of a tooth \& implant supported prosthesis.

\section{Case Report}

A 65 year old male reported with complaint of pain in the Temporo Mandibular Joint and sensitivity in multiple teeth.

On Radiological examination (Fig. 1: Orthopantomogram), it was noted that the complete occlusal load was concentrated on the bulky prosthesis present on the root stumps in the region of 46. Most of the remaining teeth required endodontic treatment before the final treatment plan for the prosthesis could be made.

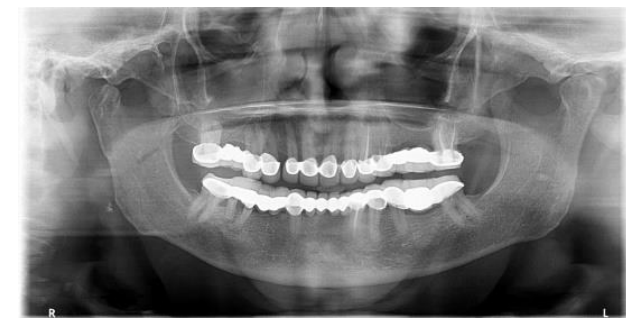

Fig. 1: Pre Operative OPG

The teeth present were $11,12,14,16,18,21,22,23,24$ \& 28 in the maxilla and $33,37,43,44,45 \& 47$ in the mandible.

On Clinical examination, there was malocclusion due to the previous poorly fabricated oversized fixed dental prosthesis

The periodontal condition of the remaining teeth was found to be satisfactory.

Patient was informed about the need for removal of the root stump present in the region of 46.

The advantages \& disadvantages of the different prosthetic treatment options were explained to the patient.

On CBCT (Cone Beam Computed Tomography) examination, it was noted that there was approximation of Inferior Alveolar Nerve in the region of 46. Hence, the decision to place an Implant in the region of 46 was avoided.

Since, the patient desired a long term prosthetic treatment, it was planned to retain the periodontally sound teeth and endodontically treat the remaining natural teeth before giving a fixed dental prosthesis.

Dental Implant (Equinox Myriad - $4.5 \mathrm{~mm}$ x 11.5 $\mathrm{mm}$ ) (Fig. 2) was placed in the region of 44 and a combination of a tooth and Implant supported prosthesis was planned to replace missing teeth in the region of 44 and 45 to avoid demerits of long span prosthesis. 


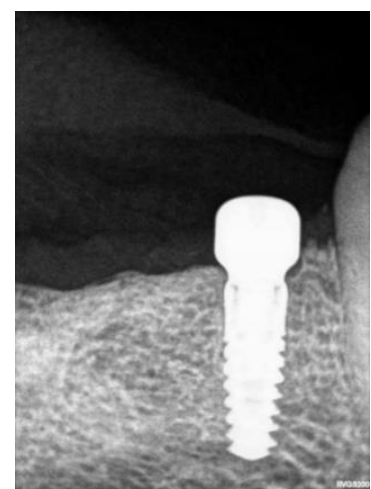

Fig. 2: Radiograph after implant placement

The prosthetic phase was initiated 4 months after the placement of the Implant and the prosthetic rehabilitation was initiated.

The impression of the teeth and implants were made in Additional silicone (Aquasil, Dentsply) and the standard laboratory procedures were carried out for the fabrication of the prosthesis.

A 4 unit prosthesis was fabricated that involved the support of implant in the region of 44 and natural tooth in the region of 47 and tooth supported in the region of 35 38

A 7 unit fixed dental prosthesis was fabricated from 43 to 34 to replace the lower anterior teeth.

A 3 unit fixed dental prosthesis was fabricated from 16 to 18 .

Upper anterior teeth were individually crowned and a 4 unit prosthesis was fabricated from 24 to 27 .

Patient was instructed to maintain proper oral hygiene and report after 1 week for review and the recall visits were scheduled after 1 month, 3 months followed by every 6 months.

During each visit the oral hygiene maintenance, periodontal health status and fit of the prosthesis were assessed.

On each visit, an Intra Oral Periapical Radiograph was taken to notice any resorption of bone around the dental implant i.e. After 1 year (Fig. 3) and After 2 years (Fig. 4).

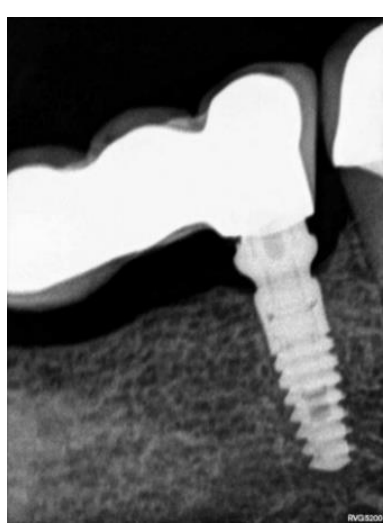

Fig. 3: 1 Year Follow up Radiograph

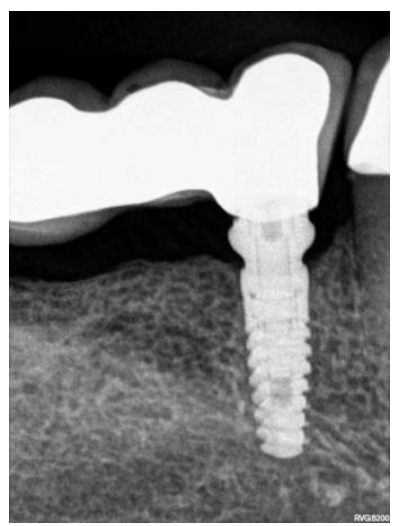

Fig. 4: 2 Year Follow up Radiograph

A 3 year follow up showed no crestal bone loss around the region of the Implant. (Fig. 5)

The patient is comfortable with his new prosthesis.

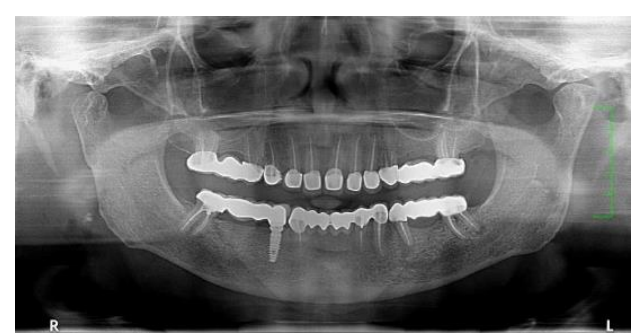

Fig. 5: Post Operative OPG

\section{Discussion}

A tooth implant combination prosthesis is supported by both dental implant and natural teeth. The distribution of remaining natural teeth in certain cases may not be favourable for the ideal fixed dental prosthesis. Hence, they are supplemented with dental implant as the last resort. Providing a combined prosthesis not only provide numerous advantages like additional support, proprioception, better load sharing but also reduces the financial burden on the patient.

The potential risk involved in combination prosthesis is the difference in the biomechanics of the prosthesis supported by the natural teeth and the dental implants.

In cases of prosthesis supported by natural teeth, the periodontal ligament transfer forces to the surrounding bone whereas in an implant the concentration of the force is at the crestal bone level.

It is necessary to provide an implant protected occlusion in such scenarios to increase the long term prognosis.

\section{Conclusion}

A 65 year old patient's dentition was successfully restored by using a combination prosthesis supported by both, a dental implant and natural teeth. A 3 Year Follow up revealed, no to negligible bone loss (Under Physiological Limits) at the crestal level surrounding the implant suggestive of equal distribution of load to 
the combined natural tooth and the implant. Proper designing \& execution of the dental prosthesis can help the clinician overcome the problem associated with stress distribution.

Funding: No funding sources.

Conflict of interest: None declared.

\section{References}

1. Hobo S, Ichida E, Gacia LT. Osseointegration and occlusal rehabilitation. Tokyo: Quintessence; 1989.

2. Carl E Misch. Contemporary Implant Dentistry. 3rd ed. Elsevier; 2007.

3. Lang N, Pjetursson B, Tan K. A systematic review of the survival and complication rates of fixed partial dentures after an observation period of atleast 5 years, combined tooth implant supported FPDs. Clin Oral Implants Res 2004;15(6):643-53.

4. Brunski JB, Puleo DA, Nanci A. Biomaterials and Biomechanics of oral/maxillofacial implants: Current status and future developments. Int J Oral Maxillofac Implants 2000;15(1):15-46.

5. Palmer RM, Howe LC. Implant dentistry-prosthodontics: a prospective 3 year study of fixed bridges linking Astra tech ST implants to natural teeth. Clin Oral Implants Res 2005;16(3):302-7.

6. El Charkawi Hussein G, Zerky Khaled A, Mohammed T.Elwakad. Stress analysis of different osseointegrated implants supporting a distal extension prosthesis. $J$ Prosthet Dent 1994;72(6):614-22.

7. Clelland NL, Ismail YH, Zaki HS, Pipko D. Three dimensional finite element stress analysis in and around the screw and implant. Int J Oral Maxillofac Implants 1991;6(4):391-8.

How to cite the article: Anand D., Sharma R., Yadav D. Full mouth rehabilitation with tooth implant connection prosthesis: A case report with follow up. IP Int $J$ Periodontol Implantol 2018;3(3):115-117. 